\title{
La Démocratie technique au prisme des Sciences
} sociales

\author{
Technical democracy through the lens of social sciences
}

\author{
Jean-Claude Ruano-Borbalan ${ }^{1}$ \\ ${ }^{1}$ Laboratoire HT2S, Conservatoire National des Arts et Métiers, France, jean-claude.ruanoborbalan@lecnam.net
}

\begin{abstract}
RÉSUMÉ. Cet article s'interroge sur l'écart insurmontable existant entre un idéal démocratique d'implication et de participation citoyenne, exprimé dans des formes contemporaines, et la réalité de biais et contraintes fortes pesant sur les processus et dispositifs de participation et délibération, notamment et principalement dans le cas des rapports technoscience et société, communément désignés sous le vocable de "démocratie technique». II utilise pour en penser l'articulation et les éventuels dépassements, les apports récents des sciences sociales et humaines, notamment des sciences et théories de l'organisation.

ABSTRACT. This article focuses on the one hand, on the insurmountable existing difference between a democratic ideal which emphasizes citizen empowerment (in the contemporary deliberative and participatory approaches) and on the other hand, upon the existence of dramatic bias and constraints (cognitive and sociological mainly, but also political, institutional norms, etc.). It uses social sciences, psychology and notably organizational theory to grasp the understanding of problem, and possibly give some ways to encompass the huge contradictions.

MOTS-CLÉS. Participation, sciences et démocratie, démocratie technique, technique, théorie des organisations, collaboration, biais cognitifs.

KEYWORDS. Participation, sciences and democracy, technical democracy, sociology of organizations, collaboration, cognitive bias.
\end{abstract}

La tradition démocratique occidentale se nourrit d'un postulat dominant du sujet libre doté de raison. L'économie politique, à partir du XVIIIème siècle, a ajouté que ce sujet serait à la fois " utilitariste» et rationnel. La littérature des sciences sociales et de l'économie, fortement renforcée par le développement des sciences cognitives, de la psychologie sociale et des diverses approches constructivistes ont depuis plusieurs décennies remis en cause la fiction du sujet purement rationnel. [DUP97]

L'individu n'est pourtant ni libre, ni rationnel, ou du moins use-t-il d'une rationalité «limitée» comme l'ont montré et admis successivement les sciences sociales et la psychologie cognitive. L'individu en société, qu'on le pense acteur social, sujet ou agent, selon les points de vue disciplinaires n'est même probablement pas précisément «utilitariste», au sens ou il tenterait en permanence de maximiser ses intérêts, quoi qu'il faille probablement admettre que ses motivations multiples, souvent disjointes voire contradictoires, sont liées aux tentatives de réaliser des «gains» psychiques, symboliques ou matériels. La littérature contemporaine, immensément abondante et évidemment contradictoire, s'accorde majoritairement sur ces points. [COL11]

L'une des questions les plus prégnante aujourd'hui tourne autour de la «démocratie technique», vocable très prisé et développé en France à partir des travaux d'analyse des rapports sciences, techniques et sociétés (notamment ceux de Michel Callon et Bruno Latour) qui se sont singulièrement modifiés à partir des années 1970-1980 [LEQ14]. La «démocratie technique», vue comme l'émergence et le renforcement de mobilisations face aux conséquences des «technosciences» recouvre plusieurs interrogations et traditions. Elle ressortit au moins, de la cristallisation de trois traditions politiques ou intellectuelles majeures :

- La longue, et fort conflictuelle, tradition de pensée de la démocratie occidentale et ses interrogations sur les formes de la représentation, de la délibération citoyenne. 
- La nature et l'histoire des sciences, des techniques et de l'innovation industrielle. Et donc l'analyse des rapports entre les sociétés et le progrès ou les dégats de la civilisation industrielle et technoscientifique.

- Les formes de mobilisation sociales et politiques qui se sont fortement transformées dans les années 70 à 90 du siècle dernier.

On peut admettre que les débats contemporains sur la démocratie technique, si multiples, intègrent des préoccupations (et des acteurs) issus de ces trois traditions ou secteur d'action politique. Ce constat permet d'interpréter l'existence de contradictions majeures au sein des discours et pratiques s'en réclamant, comme l'a pointé par exemple Jean Paul Gaudillère en 2003, dans un compte rendu du livre pinceps de M.Callon, P.Lascoumes et Y.Barthes, en notant que la vision «dialogique» des auteurs du livre «Agir dans un monde incertain» pouvait être disqualifiante aux yeux de ceux qui «à gauche», $\mathrm{y}$ verraient une sorte de masque à des réalités de domination, de pouvoir direct, bref de «luttes» plutôt que de «co-construction». [GAU02]

\section{Sciences et démocratie, quels rapports?}

Quinze années plus tard, les traditions se sont singulièrement mixées et le paysage interprétatif et d'action s'est reconfiguré. Si la question de la démocratie technique et de l'analyse des mobilisations ou actions autour des conflits ou controverses technoscientifiques demeurent, elles sont plus ou moins intégrées à une vaste question : les rapports entre sciences et démocratie, voire sciences et sociétés. Plusieurs raisons à cela, sans qu'aucune ne puisse être conçue comme prioritaire :

D'abord la transformation spectaculaire des systèmes productifs tant dans leur géographie mondiale que dans leurs formes et réalités de production ou circulation de biens et services. Il est ici hors de propos d'en faire une analyse qu'on pourra trouver dans de multiples travaux, notamment de géographie politique et économique [BRE13], mais notons tout de même la modification liée aux technosciences, notamment -mais pas seulement- celles qui touchent à la communication, aux réseaux, pour comprendre leur impact sur les pratiques de concertation (discussion, délibération) et mobilisations sociales des sociétés contemporaines.

Mais aussi le renforcement de plus en plus net d'une compréhension constructiviste de la conflictualité organisationnelle ou sociale : les questions de pouvoir que l'on pouvait concevoir comme descendantes et coercitives, sont pour l'essentiel aujourd'hui vues comme des questions d'interactions multiples ou les formes d'autorité, de domination ou de sujetion relèvent d'une compréhension relationnelle, notamment au prisme des réflexions de Michel Foucault qui a porté l'emphase sur le rôle des discours et des normes ou institutions dans les processus d'action collective ou individuelle, des définitions mises en avant par les sciences politiques ou la sociologie mettant en avant les relations et les stratégies d'acteurs (Robert Dahl; Michel Crozier) ou la psychologie sociale cognitive (mise en avant des questions identitaires ou affectives).

Ensuite et précisément, autour technosciences, dont le système de production s'est hypertrophié et renouvelé, comme le montre les études en sciences sociales des sciences, des techniques, qui notent d'une part que le régime dominant de production de la connaissance légitime est entièrement scientifique (avec des mutations de formes et de nature des disciplines) et d'autre part que l'autorité et l'expertise scientifique se sont affirmées dans les principales sphères de l'action politique ou économique. [PES10] De ce point de vue, les hypothèses de défiance vis-à-vis de la science ne sont pas avérées, au contraire. Si les individus et collectifs des sociétés contemporaines sont attentifs et débattent nettement des risques (produits de la science et de l'activité industrielle liée), s'ils ne sont plus du tout cadrés - comme au XIXème et XXème siècle par des visions d'un progrès linéaire et sans discussion des sciences, ils ne sont pas pour autant défiants vis-à-vis de la technoscience et de ses résultats ou production, comme par exemple les avancées médicales ou l'utilisation de postures 
d'actions politiques et administratives en matière éducatives, de plus en plus inspirées par des fondements et expertises scientifiques de sciences cognitives, etc.

Enfin autour de l'émergence spectaculaire de visions en terme d'innovation et de «design» qui se sont emparée de toutes les sphères d'activité humaine, jusqu'à devenir un discours ubiquitaire et l'idéologie positive des «elites» politico-administratives et économiques de l'ensemble de la planète. [BER17]

Si l'on reste aux questions de démocratie -technique ou non- le constat est singulier: on a vu converger depuis ce qu'il est convenu d'appeler le «deliberative turn» [DRYO3] de la fin du XXème siècle, des traditions libérales, anarcho-syndicalistes, libertaires, mais aussi...d'innovation entrepreneuriales ou associatives fondées sur le rapport entre projet et technologies de fabrication individualisées, d'innovation et de rationalisation administrative, de participation des usagers et citoyens dans la «fabrique des territoires», etc. Les préoccupations et affirmations de démocratie technique se sont modifiées. On s'interroge, selon l'origine de la réflexion: sur la possibilité de développer des sciences participatives; sur les questions de «transfert» de connaissance pour l'innovation techno-industrielle; sur les questions de risques et de maitrise du futur (notamment autour de la question climatique ou environnementale) ; sur les possibilités et rôle de l'expertise scientifique ; sur les potentialités de l'innovation sociale; sur la «maitrise d'usage» dans les problématiques urbaines; sur le design des formes collaboratives en organisation ou dans le cadre de politiques publiques, etc. Cette dilution et éclatement des questionnements peut être vue comme un affadissement. Nous le tenons pour signe du succès de l'interrogation portée autour de la démocratie technique quelques décennies plus tôt.

En effet, si l'on prend pour exemple la question de la «participation» et les possibilités de la délibération politique au plus près des «citoyens-usagers-clients», qui constitue l'objet même du présent numéro de la revue Technologie et innovation, on voit qu'elle peut être reliée, à l'histoire de la pensée politique de la démocratie, mais aussi aux évolutions des sciences sociales (la sociologie pragmatique, la linguistique et les sciences de l'information et de la communication, ou les sciences de l'apprentissage coopératif notamment. Elle concentre les possibilités de compréhension de la transformation démocratique elle-même, à l'ère technoscientifique. Les interrogations anciennes sur la place et la limitation de l'intervention citoyenne dans la marche de la gouvernance et de l'action politique, sont par exemple renouvelées par des approches centrées sur les capacités d'intervention et d'innovation sociale. Même si, on constate des limites nettes de ces capacités d'interventions, par exemple dans les politiques et pratiques de l'urbanisme, qui pour autant s'en gargarisent au plan du discours.

La question générale posée est aujourd'hui de savoir s'il est possible (et si oui comment) d'intégrer dans une visée d'action politique quelle qu'elle soit- à la fois un idéal démocratique renouvelé et les biais et obstacles anthropo-sociologiques et psychologique incontournables qui font le comportement et les interactions des membres de sociétés contemporaines, comme d'ailleurs les organisations ou institutions qui les composent? En d'autres termes, de dépasser la contradiction entre un idéal de liberté et d'égalité des individus en situation de délibération démocratique et une réalité contrastée et discontinue, faite de biais multiples: croyances, intérêts, conflits d'autorité, raisonnements contradictoires et construction des arguments utilisés?

\section{La participation citoyenne : nécessité ou/et impossibilités}

Il n'est, on s'en doute, pas simple, ni même peut-être possible de répondre à de telles questions. Du moins peut-on tenter de chercher au sein des sciences sociales et des sciences humaines des éléments de réponses, et constater que les deux aspects (l'idéal et la réalité) sont deux face d'une même pièce. 
Le philosophe James Bohman estime par exemple que les sociétés démocratiques contemporaines sont désormais conduites par " nécessité» à la mise en œuvre de formes de délibération en raison de la complexité même de ces sociétés (individualistes, pluralistes, réflexives et médiatiques, etc). [BOHO6] Si la thèse est discutable, elle est désormais relativement partagée. [THO08] Cependant, et c'est James Bohman lui-même qui le rappelle, il existe un «soupçon» fort, il parle de «scepticisme sociologique» sur la place et l'intérêt d'un approfondissement délibératif à nos formes démocratiques. Le premier volet de ce soupçon est formulé par la sociologie politique, et l'on se reportera ici à l'argument complet et fort de Loïc Blondiaux. [BLO08] Ce dernier porte sur la réalité même des expériences concrètes de délibération/participation citoyennes. Il souligne que celles-ci sont de piètre ampleur en réalité : même pour l'expérience de budget participatif à Porto Allegre, vantée en son temps comme une réussite dans nombre de cénacles, ce ne sont que 1 à $2 \%$ de la population qui ont été impliqués, avant l'épuisement de la formule. Les formes de délibération actuellement utilisées sont presque totalement prisonnières des institutions politiques existantes. Elles ne portent pour l'essentiel pas sur des problèmes d'ordre généraux permettant aux individus de s'exprimer en tant que «citoyen global». Elles privilégient dans la plupart des cas l'expression des couches favorisées. Et, last but not least, elles n'ont, pour l'essentiel- en tout cas en France-, aucun moyen réel de peser sur les décisions politiques.

Plus généralement, le «scepticisme» concernant la délibération (capacité coopérative et argumentation) est lié aux analyses et réflexions que mènent depuis plus d'un siècle les sciences humaines et sociales sur les processus de constitution et de vie des groupes et la cognition, qu'elle soit sociale ou individuelle. La psychologie, la sociologie, l'anthropologie et plus récemment les sciences de la communication, ont notamment démontré, et démontrent chaque jour un peu plus, que les individus ont une cognition et des rapports sociaux cadrés et « sur-déterminés». Non par la valeur des arguments comme on le pense spontanément dans la tradition politico-philosophique occidentale, ou même par l'exercice démocratique du pouvoir, mais cadré par des contraintes psycho-affectives ou des contraintes spécifiques, liées à l'interaction et la communication sociale. En d'autres termes, les individus ne sont pas les sujets libres argumentant raisonnablement, avec une excellente capacité d'expression de leurs jugements, mais au contraire des individus soumis aux contraintes de leur cognition, au prisme de leurs émotions, aux contraintes linguistiques.... Tous ces biais «internes» de la cognition humaine s'expriment dans le cadres d'interactions de communication et au sein de réalités, normes et dispositifs sociaux, organisationnels ou institutionnels. Cet ensemble de contraintes constitue une limitation extrêmement importante de leur capacité rationnelle d'argumentation et de décision. [BOU07]

Nous privilégierons ici une approche issue de la réflexion sur les organisations -l'une des branche importante des sciences sociales et sciences humaines-, car elle peut constituer un outil très important pour penser les mécanismes et biais à l'œuvre dans les processus délibératif/participatifs, et éventuellement en dépasser les contradictions. En effet la délibération dans ses formes réelles utilisées (budget participatif ; jurys citoyens ; conférences de consensus ; enquête publiques) peut être conçue comme un dispositif organisationnel spécifique. Le recours une analyse de la délibération/participation comme processus organisationnel est validé par la nature même des processus en cause. Les dispositifs utilisés ont un caractère pérenne, structuré, avec une logistique et une stratégie parfois très lourde, qui en font de manière assez évidente des organisations embryonnaires, voire des organisations tout court. On peut ajouter que ce sont des organisations administratives et politiques, qui cadrent et mettent en œuvre les formes de la délibération citoyenne, notamment dans le cadre de la démocratie technique. Ces organisations, nourries de leurs cultures et pratiques propres, implicitement ou explicitement analysées, transmettent et formatent les dispositifs de participation.

\section{Une théorie de l'action collective pour penser la participation/délibération ?}

La théorie des organisations dont nous entendons utiliser les apports est pour une part une théorie de l'action collective. L'action collective comporte deux versants majeurs qui se retrouvent totalement 
dans les processus politiques d'engagement citoyens : l'acteur (le citoyen) et le système (le dispositif précis; les institutions et le cadre politique, etc). Si, comme on le sait, la tradition occidentale de philosophie politique, d'économie politique et de théorie politique pensent prioritairement le citoyen comme rationnel et capable d'une argumentation (elle aussi rationnelle), la théorie des organisations a montré, que l'individu est avant tout «stratégique» [CRO77] : il poursuit des buts de maîtrise et de pouvoir, sur les choses et sur autrui, dans des situations ou l'information est incomplète et surtout avec une rationalité «limitée». [ALL71]

Il faut noter, et c'est essentiel pour une réflexion à visée de compréhension de formes démocratiques, qu'en contrepoint de cette vision critique des sciences sociales et de l'organisation s'est développé une considérable littérature managériale, liée mais non confondue avec la théorie des organisations. Dans ces réflexions, fortement influencées par les découvertes de la psychologie ou de la systémique, l'engagement et la motivation (l'apprentissage) des individus ont été progressivement conçus comme l'une des clef principale de la performance collective. La littérature managériale a popularisé, dans les quarante dernières années, l'idée que la participation des salariés est cruciale. On a donc, notamment dans les domaines de la formation et des ressources humaines développé des dispositifs et une ingénierie considérable (les principales tournent autour du concept «d'organisation apprenante») pour le permettre... avec un succès souvent relatif. [BOU09] C'est la question de la coopération en groupe (en l'occurrence en situation de travail) qui a fait l'objet de toutes les attentions, et qui pourrait être utile pour l'ingénierie des dispositifs participatifs ou délibératifs (elle l'est dans les processus de sondage délibératif par exemple).

\section{La délibération/participation comme forme de la coopération}

La question de la coopération, si prégnante pour notre objet, a été conceptualisée principalement par la psychologie cognitive [STE04], la psychologie sociale [LIV08] et la science politique. [AXE84] Elle a été reprise au plan de la réflexion organisationnelle comme l'un des moyens, si ce n'est «le» moyen, d'accompagner les changements liées à la fin du fordisme et de l'organisation taylorienne du travail. La coopération en organisation s'incarne dans la coordination de tâches, d'activités, d'équipes ou de personnes, désormais vue sous un angle positif, valorisant la «libération» des organisations. [PAT17]

Malgré les transformations techno-organisationnelles contemporaines, notamment les nouvelles technologies de la communication et des réseaux, le rêve d'une coopération et d'un engagement permanent des acteurs en situation de collaboration organisationnelle (Disons le de nouveau : les processus de participation et délibération peuvent s'y apparenter), est illusoire. Si l'on suit les travaux des sciences sociales (et de la psychologie) contemporaine, on sait que les acteurs sociaux se servent de l'information dont ils disposent à des fins de préservation de pouvoir ou de conflit, et ne sont surtout pas enclins à coopérer directement, spontanément et en toute transparence. [CRO77] Tout au contraire, ils savent le prix des informations dont ils disposent. Ils savent que la résistance passive est un mode d'action puissant, qu'il ne convient pas d'argumenter en toute circonstances, etc.

Ces remarques valent pour tout humain engagé dans l'action, y compris dans un processus de concertation et de délibération citoyenne bien entendu. Il est notamment probable que la sousreprésentation chronique des classes populaires ou immigrés [STA14] dans les instances délibératives locales soit liée à la conscience de l'inutilité prévisible de leurs argumentations, compte tenu des préjugés (bien connus de ceux qui les subissent) que les populations des classes moyennes et supérieures entretiennent vis à vis de membre de ces couches sociales.

La vision actuelle de la collaboration et de l'action collective privilégie les analyses en termes de réseaux. [LAL15] Elle souligne le fait qu'un individu n'entre pas «vierge», muni de sa seule capacité argumentative ou raisonnante, dans la relation interpersonnelle ou l'action (et la délibération citoyenne relève à la fois de la relation, de la communication et de l'action). Cette question des réseaux est commune à l'analyse de l'espace politique : on connaît les travaux de Robert Putnam sur la place du 
capital social et des réseaux dans l'engagement civique. [PUT00] Son constat de déclin des réseaux et de l'engagement politique (constat fortement discuté d'ailleurs depuis) fournit une possible explication à la désaffection du politique tant redoutée. Les réseaux constituent, dans cette vision désormais commune en termes de «capital social», de puissants moyens de protection et de fiabilisation de l'action pour les individus, qui agissent et s'expriment en référence à leurs cadres d'appartenance. De ce fait, les réseaux jouent un rôle majeur de contrôle social et de définition du pouvoir. [MER16]

On peut émettre l'hypothèse que, dans les processus de coopération délibérative ou de participation, l'individu définit son argumentation non seulement en fonction de la situation présente, de la rationalité argumentative ou de l'information dont il dispose sur le moment, mais en lien avec l'image qu'il a de ses contraintes de réseaux : que diraient mes relations, ma communauté, mon groupe d'appartenance? Une telle approche relève de la compréhension de l'identité (self) et l'estime de soi qui sont en jeu dans ce type de situations. [RUA03] La construction identitaire en organisation et au travail est une bonne approche de ce point de vue, car elle s'effectue dans un cadre contractualisé et n'engage pas complètement l'individu, comme dans une situation de participation ou de délibération citoyenne.

\section{La délibération/participation comme processus cognitif}

Du point de vue de l'action collective, et tout particulièrement de l'analyse des organisations, on s'est mis à scruter les relations humaines, les motivations et la cognition en situation collective dès la première moitié du XXème siècle. [ROJ03] Au sortir de la seconde guerre mondiale, les sciences sociales et la psychologie ont procédé la réévaluation du rôle des individus, de leurs affects (travaux d'Eliot Jacques notamment). C'est à partir des travaux de Chester Barnard, mais surtout de James March et Herbert Simon, repris en France par Michel Crozier et bien d'autres, que la question du rapport entre les acteurs et l'organisation (conçue comme un système coopératif singulier organisé en système, avec règles et contrats) s'est développée. [ROT16]

Cette préoccupation s'est affirmée dans les dernières décennies, en lien notamment avec la mutation des systèmes productifs qui s'appuient sur des sociétés dites de la connaissance, où « l'apprentissage intensif» est valorisé. Plusieurs directions ont été explorées et développées, tant du point de vue de l'organisation, que du point de vue de la cognition individuelle. Les recherches sur la connaissance dans les organisations « ont tendu à se structurer en deux familles d'approches : celles qui, partant de l'activité des acteurs individuels, cherchent à intégrer le fait social comme une donnée essentielle de cette activité ; celles qui, partant de l'étude des organisations comme structure d'action collective, cherchent à intégrer la dynamique d'apprentissage des acteurs comme donnée essentielle du fonctionnement organisationnel. [LOR14] [TEU05]

On a vu à partir des années 70 sous l'impulsion des travaux de Chrys Argyris et Donald Shön, la cristallisation d'une approche de l'organisation et du changement organisationnel en terme «d'apprentissage» collectif dit « learning organisation». Cette approche a eu une ascension foudroyante et une extension considérable [EAS15], notamment à partir du livre de l'ingénieur, professeur au MIT, Peter Senge [SEN90], qui en 1990 a formalisé dans un langage accessible une doctrine managériale fondée sur l'apprentissage organisationnel. Il est de fait que les organisations avaient à faire face des environnements devenus progressivement de plus en plus concurrentiels, que le niveau de scolarisation et de réflexivité des individus s'accroissait, et que la vision systémique invite à penser l'organisation comme un «individu», capable d'apprentissage. Le concept d'organisation apprenante est apparu dans un contexte particulier: société du savoir, technologies de l'information et des communications, obsolescence et explosion des connaissances, changements dans le monde du travail, apprentissage tout au long de la vie, etc. [BOU14]

L'intérêt principal de la réflexion en terme d'organisation apprenante pour des processus d'action collective et donc de délibération/participation peut résider dans l'affirmation que les organisations et 
les systèmes institutionnels apprenants sont ouverts. Ainsi, des pratiques de stockage de l'information et de définition «culturelle», de cadrages et de «design» des formes de la coopération et de la décision sont nécessaire pour qu'un apprentissage collectif ait lieu...et dure dans quelque cadre institutionnel ou organisationnel que ce soit, y compris dans des formes associatives embryonnaires que peuvent être les processus de participation. [FAB11] Par analogie, il paraît raisonnable de penser que les dispositifs techno-informationnels, désormais généralisés, sont intéressants pour la réflexion touchant aux formes de la démocratie technique et de la participation/délibération correspondante, [CAR12] même si initialement, au sein des sciences sociales on s'est plutôt méfié du rôle des réseaux sociaux, comme l'illustre le propos suivant de Partice Flichy : "internet n'a pas en lui-même d'effet négatif sur la délibération démocratique. Il s'est en partie moulé sur les caractéristiques de notre société, mais il offre aussi de réelles opportunités pour de nouvelles formes démocratiques, multiples et réticulaires, où le citoyen ne se contente pas d'élire ses représentants, mais où il peut débattre, surveiller et évaluer leurs actions.» [FLY08]

Pour autant, la réflexion sur l'apprentissage organisationnel, si pregnante dans les années 90 , s'est vu complétée par la convergence des multiples réflexions sur la cognition « en situation» autrement nommée dans les sciences de la formation «savoirs d'action», qui sont à la fois plus générales et plus adaptées à tout les types de pratiques et de réalités de l'action collective et de la collaboration. [GAL06]

Dans le vaste océan des réflexions de sciences cognitives, la réflexion sur la cognition en action, située ou distribuée a pris de l'importance pour l'analyse des formes de coopération, notamment (mais pas seulement) en situation de travail. Cette réflexion sur la pratique et les communautés de pratiques est issue fondamentalement de l'essor des réflexions de sciences de gestion et surtout de l'analyse des systèmes d'information et d'ingénierie des connaissances : une activité considérable que les nouvelles technologies informatiques et d'intelligence artificielle ont générées. Dans cette perspective d'ingénierie des connaissances le but fondamental des scientifiques et ingénieurs fut et demeure modéliser les apprentissages individuels (le modèle cybernétique et l'intelligence artificielle) ou «en situation». La nécessité de formaliser la connaissance pour bâtir des systèmes informatiques experts a conduit progressivement à une rencontre entre les sciences de l'informatique et de l'intelligence artificielle d'une part et les analyses des pratiques professionnelles ou sociales (psychologie du travail et de l'activité ; ergonomie ; sciences de l'information et de la communication ; etc) d'autre part. Le paradigme dominant des ingénieurs et informaticiens, à savoir la conception du cerveau humain comme une machine informatique, que l'on pourrait in fine reproduire a dans le même temps trouvé ses limites, dans les avancées conceptuelles des sciences cognitives. [CHI17] L'une des conséquences de cet épuisement de la tentative « techno-scientifique» de recréation du cerveau humain par des voies informatiques a été, dans les sciences de gestion, de l'information et de l'éducation/formation, le déplacement de l'étude des mécanismes cognitifs internes du sujet/acteur vers de l'étude du couplage activité/situation. [GRI04] En effet, on a revalorisé les études de la cognition privilégiant l'environnement et ses contraintes sociales, de communication ou de dispositif, notamment en retrouvant et réévaluant des travaux pionniers, comme ceux de Lev Vigotski (psychologue russe des années 30) ou de Jérôme Bruner, de Harvard, qui le premier dans les années 60 avait souligné le fait. Indépendamment des filiations et combats entre écoles d'interprétations qui sont toujours en cours, il convient de noter qu'il y a dans ce débat sur la cognition en situation, des outils puissants pour interpréter la place et le rôle du contexte pour la formation de l'opinion et du jugement.

On doit ajouter que, pour les organisations ou les processus délibératifs/participatifs, le moment est probablement particulièrement propice à une réflexion privilégiant des processus transversaux et collaboratifs. Il y a à cela deux raisons: d'une part l'affaiblissement des formes du pouvoir hiérarchique (que l'on constate à tous les niveaux de l'analyse sociale : groupe, organisations, société) [RUA02] et d'autre part la généralisation des systèmes d'information et d'intelligence/savoir distribué (Ingénierie des connaissances); TIC. On constate de plus, une reprise et vulgarisation importante de ces approches dans la littérature managériale, et désormais par l'ensemble des instruments de diffusion de masse des idées et concepts : médias et éducation. 


\section{Conclusion}

Nous avons posé la question de savoir si l'on peut penser une réduction des contradictions intrinsèques aux processus de coopération délibérative et de participation citoyenne. Nous nous sommes interrogés notamment sur la réalité des biais et contraintes de la délibération et de l'action collaborative. Demeure le problème de pouvoir ou non dépasser la tension entre un idéal de liberté et d'égalité des individus en situation de délibération démocratique et une réalité contradictoire, où les individus défendent leurs intérêts, argumentent de manière discontinue et contradictoires, où les questions de pouvoir et de croyances sont totalement prégnantes, etc.

Cette question, nous l'avons dit, traverse en premier lieu les interrogation des avatars de la «démocratie technique» que sont les sciences participatives et les pratiques ou politiques de concertation, ainsi que - dans un autre registre, proche mains non identique- au sein des discours et pratiques de l'innovation sociale. La question est ainsi, pour les rapports sciences et société, de prendre en compte l'ensemble des contraintes pesant sur les formes et normes d'action participatives : le développement industriel, les mutations du pouvoir, la domination, les conflits, les replis identitaires ou culturels, des idéologies et doctrines closes...Nous avons suggéré que les analyses, et les dispositifs opérationnels, issues des théories de l'organisation pourraient avoir une forte utilité.

Du point de vue analytique qui est ici le principal point de vue, on doit aussi et peut-être avant tout, s'inscrire dans les grandes forces à l'œuvre dans les transformations des sociétés modernes et contemporaines. Rappelons que pour penser les rapports entre sciences et société, il faut d'une part les concevoir en longue durée (au moins depuis l'avènement de ce que nous appelons la science, au XVIème siècle) et surtout inclure toute réflexion sur la participation la délibération et les rapports technoscience et société au regard de " trois dimensions essentielles de nos sociétés démocratiques et de marché : 1) l'importance des logiques marchandes, des logiques financières et de production, qui sont à la fois autonomes, transversales aux logiques dialogiques et souvent en position de forces vis-àvis d'elles; 2) l'importance des formes de gouvernement délibérément conçues, des formes de conduites dirait Foucault, qui cadrent lourdement les répertoires possible d'action, de débat et de pensée; et 3) la multiplicité des formes de régulation des produits techno-industriels, dont l'histoire et longue, les fonctions variées et les effets parfois pervers.» [PES14]

\section{Bibliographie}

[DUP97] J DUPUY J.P \& LIVET P., Les limites de la rationalité. Tome 1: Les figures du collectif. Paris: La Découverte, (2003).

[REY97] REYNAUD.B., Les limites de la rationalité. Tome 2: Rationalité, éthique et cognition. Paris: La Découverte, 1997.

[COL11] La Rationalité, Idées économiques et sociales /3 (№ 165), Editions Réseau Canopée, 80 pages, 2011.

[LEQ14] LEQUINJ.Y. \& LAMARD.P., éléments de démocratie technique, UTBM, 2014

[GAU02] GAUDILLERE.J.P., «À propos de « démocratie technique »», Mouvements, vol. no21-22, no. 3, 2002, pp. 191-193.

[BRE13] BREZNITZ.D. \& ZYSMAN.J., The Third Globalization, Can Wealthy Nations Stay Rich in the Twenty-First Century? 0xford University Press, 2013.

[PES10] PESTRE.D., Des sciences et des productions techniques depuis trente ans, Le Débat, 160, pages 115-131, 2010.

[BER17] BERTILORENZI et al., « Between Innovation and Tradition: French Design Schools, their Historical Roots and their Innovation System », Journal of Innovation Economics \& Management 2017/1 ( $\left.{ }^{\circ} 22\right)$, p. 57-78.

[DRYO3] DRYSECK.J.S., Deliberative Democracy and Beyond: Liberals, Critics, Contestations, Oxford press, 2003

[BOHO6] BOHMAN.J., Démocratie délibérative, in Le dictionnaire des sciences Humaines, dir Sylvie Mesure et Patrick Savidan, Paris, PUF, 2006. 
[THO08] THORPE.C., political theory in science and technology studies, in Edward J.Hackett \& Alii, Handbook of science and technology studies, Third edition, 2008, MIT Press.

[BLO08] BLONDIAUX.L., « Démocratie délibérative vs. démocratie agonistique ? Le statut du conflit dans les théories et les pratiques de participation contemporaines », Raisons politiques, vol. 30, no. 2, pp. 131-147, 2008.

[BOU07] BOUVIER.A., \& CONEIN.B., (ed), L'épistémologie sociale, une théorie sociale de la connaissance, éditions de l'école des hautes études, 2007.

[CRO77] CROZIER.M., \& FRIEDBERG.E., l'acteur et le système, les contraintes de l'action collective, Le seuil, Paris, 1977.

[ALL71] ALLISON.G., the essence of decision, explaining the Cuban missile crisis, Harper Collins, 1971.

[BOU09] BOURGEOIS.E., \& Alii, (dir), l’Encyclopédie de la formation, Editions PUF, Paris, 2009.

[STE04] STEVENS.J.R. \& HAUSER.M.D., Why be nice ? «Psychological constraints on the evolution of cooperation», in trends in : Cognitive sciences, harvard Press, 2004.

[LIV08] LIVET.P., «la cognition sociale et son envers», in : Déterminismes et complexité, du physique à l'éthique, autour d'Henri Atlan, sous la direction de BOURGINE.P., \& Alii, La découverte, Paris, 2008.

[AXE84] AXELROD.R., The evolution of cooperation, basic books, New York, 1984. (revised edition 2006).

[PAT17] PATRICK.G., \& Alii, «L'entreprise libérée »: analyse de la diffusion d'un modèle managérial», Revue internationale de psychosociologie et de gestion des comportements organisationnels, vol. vol. xxiii, no. 56, 2017, pp. 205-224.

[STA14] STAVAU-DEBAUGE.J., « L'idéal participatif ébranlé par l'accueil de l'étranger. L'hospitalité et l'appartenance en tension dans une communauté militante », Participations 2014/2 (No 9), p. 37-70

[LAL15] LALLEMENT.M., l'âge du faire, hacking, travail, anarchie, Le seuil, 2015.

[PUT00] PUTNAM.R., Bowling Alone: The Collapse and Revival of American Community, Simon \& Schuster, 2000.

[MER16] MERCKLE.P., Sociologie des réseaux sociaux, La découverte, 2016. (1 $1^{\text {ère }}$ édition 2006).

[RUA03] RRUANO-BORBALAN.J.C., Identité, Identités, individus, groupes, organisations, Editions Sciences Humaines, 2003.

[ROJ03] ROJOT.J.,, théorie des organisations, Editions ESKA, Paris, 2003.

[ROT16] ROT.G \&SEGRESTIN.D., Cinquante ans de sociologie des organisations Entreprises et histoire2016/3 ( $\left.\mathrm{n}^{\circ} 84\right)$.

[TEU05] TEULIER.V., \& LORINO.P, Entre connaissance et organisation, l'entreprise face au défi de la connaissance, colloque de Cerisy, La découverte, Paris, 2005.

[LOR14] LORINO.P., " Développement des compétences et reconstruction de l'activité collective », in Étienne

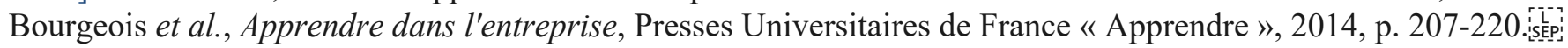

[EAS15] EASTERBY-SMITH \& LYLES.A.M., (ed), Handbook of Organizational Learning and Knowledge Management, Whyley, 2015.

[SEN90] SENGE.P., The Fifth Discipline: The art and practice of the learning organization, Doubleday, New York, 1990.

[BOU14] BOURGEOIS, É. \& ENLART, S. (2014). Apprendre dans l'entreprise. Paris: Presses Universitaires de France. doi:10.3917/puf.boug.2014.01.

[FAB11] FABBRI, T., L'apprentissage organisationnel. Dans Interpréter l'agir : un défi théorique (pp. 163-181). Paris: Presses Universitaires de France, 2011.

[FLY08] Flichy, Internet un outil de la démocratie ? LA vie des idées, Janvier 2008, http://www.laviedesidees.fr/

[CAR12] CARDON.D. \&, Marie-Carmen SMIRNELIS « La démocratie Internet. Entretien avec Dominique Cardon », Transversalités 2012/3 ( $\left.\mathrm{N}^{\circ} 123\right)$, p. 65-73.

[GAL06] GALAND.B. \& BOURGEOIS.E., (Se) motiver à apprendre. Presses Universitaires de France, 2006.

[CHI17] CHIPMAN.M.S., The Oxford Handbook of Cognitive Science, 2017. 
[GRI04] GRISON. B., Des Sciences Sociales à l'Anthropologie Cognitive. Les généalogies de la Cognition Située, @ ctivités, 1 (2), 26-34. http://www.activites.org/v1n2/grison.pdf

[RUA02] RRUANO-BORBALAN J.C., (ed), Le pouvoir, editions Sciences Humaines, Auxerre, 2002.

[PES14] PESTRE.D., «Entre hubris technologique, démocratie et choix : science, acteurs économiques, société civile», in ROSANVALLON.P., (dir) Science et démocratie, Odile Jacob, 2014. 